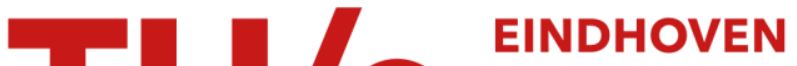 UNIVERSITY OF TECHNOLOGY
}

\section{Confinement without boundaries : Anisotropic diffusion on the surface of a cylinder}

Citation for published version (APA):

Kusters, R. P. T., Paquay, S., \& Storm, C. (2015). Confinement without boundaries : Anisotropic diffusion on the surface of a cylinder. Soft Matter, 1054-1057. https://doi.org/10.1039/C4SM02112F

DOI:

10.1039/C4SM02112F

Document status and date:

Published: 01/01/2015

Document Version:

Publisher's PDF, also known as Version of Record (includes final page, issue and volume numbers)

Please check the document version of this publication:

- A submitted manuscript is the version of the article upon submission and before peer-review. There can be important differences between the submitted version and the official published version of record. People interested in the research are advised to contact the author for the final version of the publication, or visit the $\mathrm{DOI}$ to the publisher's website.

- The final author version and the galley proof are versions of the publication after peer review.

- The final published version features the final layout of the paper including the volume, issue and page numbers.

Link to publication

\section{General rights}

Copyright and moral rights for the publications made accessible in the public portal are retained by the authors and/or other copyright owners and it is a condition of accessing publications that users recognise and abide by the legal requirements associated with these rights.

- Users may download and print one copy of any publication from the public portal for the purpose of private study or research.

- You may not further distribute the material or use it for any profit-making activity or commercial gain

- You may freely distribute the URL identifying the publication in the public portal.

If the publication is distributed under the terms of Article 25fa of the Dutch Copyright Act, indicated by the "Taverne" license above, please follow below link for the End User Agreement:

www.tue.nl/taverne

Take down policy

If you believe that this document breaches copyright please contact us at:

openaccess@tue.nl

providing details and we will investigate your claim. 
(2rossMark

Cite this: Soft Matter, 2015, 11, 1054

Accepted 2nd January 2015

DOI: $10.1039 / c 4 s m 02112 f$

www.rsc.org/softmatter

\title{
Confinement without boundaries: anisotropic diffusion on the surface of a cylinder $t$
}

\author{
Remy Kusters, ${ }^{\text {*a }}$ Stefan Paquay ${ }^{\mathrm{a}}$ and Cornelis Storm ${ }^{\mathrm{ab}}$
}

Densely packed systems of thermal particles in curved geometries are frequently encountered in biological and microfluidic systems. In 2D systems, at sufficiently high surface coverage, diffusive motion is widely known to be strongly affected by physical confinement, e.g., by the walls. In this work, we explore the effects of confinement by shape, not rigid boundaries, on the diffusion of discs by confining them to the surface of a cylinder. We find that both the magnitude and the directionality of lateral diffusion is strongly influenced by the radius of the cylinder. An anisotropy between diffusion in the longitudinal and circumferential direction of the cylinder develops. We demonstrate that the origin of this effect lies in the fact that screw-like packings of mono- and oligodisperse discs on the surface of a cylinder induce preferential collective motions in the circumferential direction, but also show that even in polydisperse systems lacking such order an intrinsic finite size confinement effect increases diffusivity in the circumferential direction.

The influence of confinement on the dynamics of glassy systems has been the focus of numerous studies on particles and polymers confined to narrow channels, or between parallel plates. ${ }^{\mathbf{1 2}}$ These works showed that the glass transition exhibits nonmonotonic behavior, depending sensitively on particle-wall interactions. $^{3-5}$ In this work, we seek to eliminate the interactions with walls to achieve a purely geometrical confinement. We do so by studying diffusion of discs on a $2 \mathrm{D}$ periodic plane. Effectively, this models the motion of discs bent onto a cylindrical surface. This way confinement without boundaries is achieved. We map out how the diffusive motion of these discs is affected by the geometry of the confinement.

The system we consider is far from academic: highly curved $^{6-9}$ and crowded motifs (see, e.g.,$^{10}$ and references therein)

${ }^{a}$ Faculteit Technische Natuurkunde, Technische Universiteit Eindhoven, 5612AZ, Eindhoven, Netherlands. E-mail: r.p.t.kusters@tue.nl

${ }^{b}$ Institute for Complex Molecular Systems, Technische Universiteit Eindhoven, 5612AZ, Eindhoven, Netherlands

$\dagger$ Electronic supplementary information (ESI) available. See DOI: $10.1039 / \mathrm{c} 4 \mathrm{sm} 02112 \mathrm{f}$ are abundant in biological and microfluidic systems. In these systems crowding and shape significantly contribute to the effective diffusivity of particles which, in the case of membraneassociated proteins, are completely confined to a curved, 2D substrate. Likewise, rapidly evolving microfluidic techniques such as topological emulsions ${ }^{6}$ and surface-confined colloids ${ }^{7}$ yield systems where crowding and curvature meet directly. Finally, the dynamics of particles in highly crowded and confined environments has been the subject of numerous studies (experimental and theoretical) in the context of flowing glassy materials, for instance in the rheological behavior of jammed emulsions in microchannels. ${ }^{\mathbf{1 1 , 1 2}}$

The effects of crowding and shape, individually, have been extensively characterized in the past: experimental and theoretical work in the context of membrane-bound diffusion have clearly established the generic effects of shape on diffusive timescales, ${ }^{8,9}$ the upshot being that shape, in itself, may alter and even direct diffusion in dilute particulate systems. The effects of pure crowding on the diffusive properties in two and three dimensions, likewise, has been widely studied (see, e.g. ${ }^{10}$ and references therein).

In the present work, we study the combined effects of crowding and confinement on the diffusion of discs bent onto a cylindrical surface. We achieve this by wrapping a 2D periodic plane with width $2 \pi d$ and length $L$ onto a cylindrical surface with radius $d$ and height $L$ Recent works have shown that at high surface coverages, the packing of discs on a cylinder is elaborately organized into various screw-like packings, where the symmetry and the tilting axes of the screw structure depend on the ratio between the radius of the crowders, $R$, and the radius of the cylinder, $d .^{\mathbf{1 3 , 1 4}}$ While this was established for athermal (granular) packings, we now measure whether these naturally arising screw-like structure alter the diffusive motion of the particles, particularly if $d$ is not significantly larger than $R$. In the following, we denote by $\zeta=\pi d / R$ the ratio of the circumference of the cylinder to twice the radius of the diffusing particles (which we fix at $R=0.5$ ). 
Using the molecular dynamics package LAMMPS, ${ }^{15}$ we execute two-dimensional Langevin dynamics simulations with a damping time $\tau=0.1$ Lennard-Jones time units (see ESI I $\dagger$ ). We point out that the Lennard-Jones interaction is evaluated in $2 \mathrm{D}$ : the separation between interacting discs is the shortest centerto-center path (i.e., the geodesic) along the curved surface, rather than the shortest 3D center-to-center path, which passes through the 3D interior of the cylinder. For membrane-bound proteins, the dominant mutual interactions are often membrane-mediated ${ }^{16-18}$ in which case the $2 \mathrm{D}$ separation is indeed the relevant one, but there may be important situations (charged proteins at small zeta, for instance) in which it is not. While we consider only cylindrical systems in this article, we note that the computational techniques to address similar problems on arbitrarily curved surfaces have very recently become available. ${ }^{19}$ We focus on the regime where the Mean Squared Displacement (MSD) scales linear with time, $\left\langle\Delta r^{2}\right\rangle=$ $4 D(\phi) t$ (see Fig. 1(b)). Here the MSD is calculated taking into account the periodicity of the system, i.e., the displacement in the circumferential direction is not bounded to $2 \pi d$. In highly crowded systems, the long-term diffusivity is dominated by global rearrangements of large clusters of particles, caused by so called cage-rearrangements. ${ }^{20}$ In Fig. 1(c) we illustrate the displacement of particles over the course of 10000 time steps, and indicate with arrows the particles that have traveled more than twice the root mean square displacement $\Delta r>2 \Delta r_{\mathrm{MSD}}$. In the narrow systems we consider, there is a natural distinction between the longitudinal and circumferential direction: the longitudinal dimension is significantly larger than the circumferential one. In Fig. 2(a), we decompose the total MSD into a longitudinal and circumferential component: $\left\langle\Delta r^{2}\right\rangle=\left\langle\Delta r_{\mathrm{C}}{ }^{2}\right\rangle+$ $\left\langle\Delta r_{\mathrm{L}}{ }^{2}\right\rangle$. This reveals that, at least for this system $(\phi=0.625$ and $\zeta=3.8$ ), the effective displacement in the longitudinal direction (a)

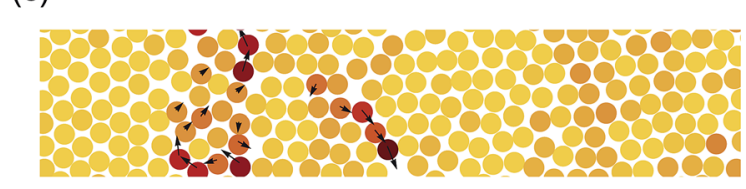

Fig. 1 (a) The system we consider: a cylinder with length $L$, circumference $2 \pi \times d$ covered with particles with radius $R$ that occupy a fraction $\phi$ of the cylinder's area. (b) The MSD $\left\langle\Delta r^{2}\right\rangle$ for various surface coverages $(\phi=0,0.5,0.625$ and 0.725 , and $\zeta=3.4)$, in the long time limit where the diffusivity is set by the collective motion. In this regime, the motion is diffusive; $\left\langle\Delta r^{2}\right\rangle=4 D(\phi) t$. In (c), red colors label the most mobile particles, and arrows indicate the displacement of the individual particle accumulated over 10000 steps (vector shown only for particles that have moved at least twice the system mean), showing the coherent motion of clusters of particles ( $\phi=0.75$ and $\zeta=7.0)$. (a) 80

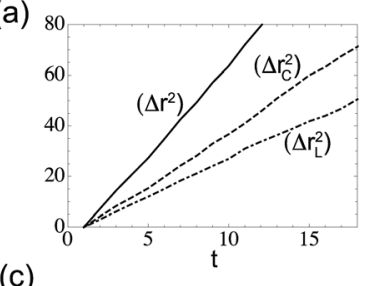

(c)
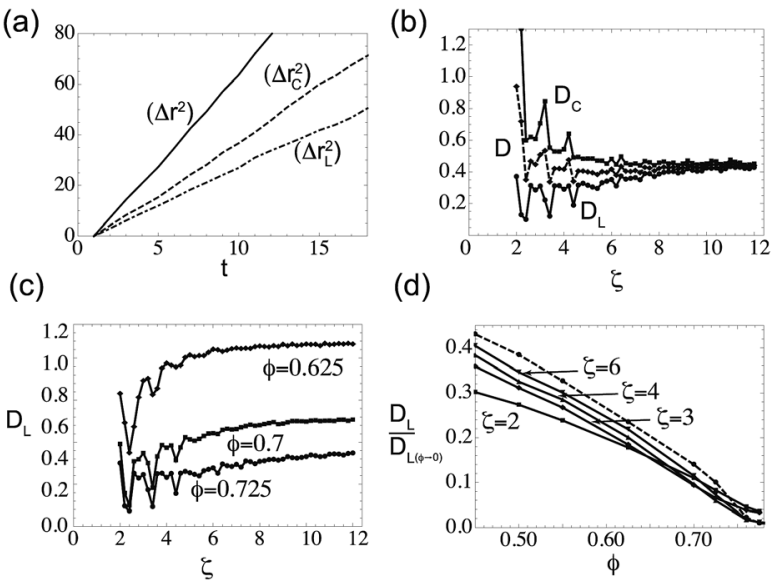

Fig. 2 (a) Time evolution of the MSD $\left\langle\Delta r^{2}\right\rangle$ and its components in the circumferential direction $\left\langle\Delta r_{C}{ }^{2}\right\rangle$ and the longitudinal direction $\left\langle\Delta r_{L}{ }^{2}\right\rangle(\zeta$ $=3.8, \phi=0.625$ and $N=2500)$. (b) shows the longitudinal $\left(D_{\mathrm{L}}\right)$, circumferential $\left(D_{C}\right)$ and total $(D)$ diffusivities as function of $\zeta(\phi=0.725$ and $N=2900$ ). (c) The longitudinal diffusivity $D_{\mathrm{L}}$ as function of $\zeta$ for various surface coverages $(\phi=0.625,0.7$ and 0.725 and corresponding $N=2500,2700$ and 2900). (d) The longitudinal component of the diffusivity $D_{L}$, relative to its value for $\phi \rightarrow 0$, as a function of the surface coverage $\phi$ for $\zeta=2,3,4$ and 6 (solid lines) and for a square $L=$ $2 \pi d$ (dashed line). The total system size was kept constant at $2 \pi d \times L=$ 1570.8 , and $\phi$ was varied by varying $N$, the number of particles in the system.

is significantly smaller than the circumferential displacement (see Fig. 2(a)).

In the following, we demonstrate that this geometrically induced anisotropy is generic in these systems. We determine the $\zeta$-dependence of the longitudinal diffusivity, $D_{\mathrm{L}}=\left\langle\Delta r_{\mathrm{L}}{ }^{2}\right\rangle / 2 t$, the circumferential diffusivity, $D_{\mathrm{C}}=\left\langle\Delta r_{\mathrm{C}}{ }^{2}\right\rangle / 2 t$, and the total diffusivity, $D=D_{\mathrm{L}}+D_{\mathrm{C}}$, for a fixed surface coverage $\phi=0.725$ (see Fig. 2(b)). We find that upon increasing the radius of the system, and thus the number of particles that will fit the circumference of the cylinder, the longitudinal diffusivity increases while the circumferential component decreases. For $\zeta \gg 10$ the two eventually become equal as the system tends to a homogeneously flat, (2D) crowded system and if $\zeta<2$ the relative position of the particles cannot exchange position in the longitudinal direction and hence $D_{\mathrm{L}} \rightarrow 0$ as the diffusion has become one dimensional. $D_{\mathrm{L}}$ and $D_{\mathrm{C}}$ are not monotonic functions; they show distinct minima and maxima. The total diffusivity $D$ also shows a non-monotonic dependence on $\zeta$, not unlike the anisotropy observed in wall confined systems. ${ }^{4,5} \mathrm{Next}$, Fig. 2(c) shows that for various values of the surface coverage, the anisotropy as a function of $\zeta$ remains. In Fig. 2(d), we show the decrease in longitudinal component $D_{\mathrm{L}}$, relative to its value for $\phi \rightarrow 0, D_{\mathrm{L}(\phi \rightarrow 0)}$, as function of $\zeta$, for various surface coverages $\phi$ and shows that the trend observed in Fig. 2(a)-(c), i.e., an increasing longitudinal diffusivity upon increasing $\zeta$, indeed appears for a broad range of surface coverages $\phi$. The dashed line corresponds to a system whose length is equal to the circumference $L=2 \pi d$ and thus exhibits isotropic diffusion. Eventually at $\phi \approx 0.82$ the diffusivity vanishes as the system becomes glassy. 
In Fig. 3(a) we calculate the ratio $D_{\mathrm{C}} / D_{\mathrm{L}}$ as a function of $\zeta$ for various surface coverages $\phi$, and find that although it shows an overall decrease with increasing $\zeta$, maxima in the anisotropy arise periodically. The period of these peaks equals $\zeta=1$ and they occur each time one additional particle precisely fits the circumference of the cylinder. The height of these peaks in $D_{\mathrm{C}} / D_{\mathrm{L}}$ decreases as a power law with an exponent of approximately 3 , though we have a limited range and cannot firmly establish this scaling. We note, too, that the magnitude of the heterogeneity decreases with decreasing $\phi$, establishing that it is truly the combination of shape and crowding, and not just the shape itself, that causes the effects we describe here.

The question we address now is whether the observed effect is a consequence of the configurational order of the particles, i.e., of the screw-like patterns that naturally arise in these dense systems. ${ }^{13,14}$ To quantify this, we apply the standard Delaunay triangulation scheme to find the lines connecting each particle to its 6 nearest neighbors. Then, we calculate the orientation (relative to the circumferential axis, see Fig. 3(b)) of these lines. To properly account for the bonds at the periodic edges of the system we also include bonds with the mirror images of the system. Next, we tabulate the distribution of these angles $\alpha$ for $\phi$ $=0.725$ at those $\zeta$ where $D_{\mathrm{C}} / D_{\mathrm{L}}$ exhibits extrema (see Fig. 3(c)). We see that the anisotropy in diffusivity is strongly correlated to the spatial configuration of the particles. Specifically, we find that the structures where the dominant orientation is in the longitudinal direction, which correspond to $\alpha=0$ and $\alpha=1.1$ as peak values, have a larger diffusivity in the circumferential direction compared to systems which have $\alpha=0$ and $\alpha=0.5$. These two states would, if densely packed, correspond to the symmetric packing and its affinely rotated structure discussed by Mughal et al. ${ }^{14}$
Our observations strongly suggests that the configurational order of the system causes the diffusive anisotropy. The longterm diffusivity in highly crowded systems is generally dominated by so called cage-rearrangements. ${ }^{20}$ We hypothesize that the cage rearrangements in these screw-like configurations induce motion in a preferred direction. To verify this, we have calculated the orientation and the magnitude of the displacement for various values of $\zeta$. As may be seen in Fig. 3(b), those particles with a displacement larger than twice the mean, $\Delta r>2$ $\times \Delta r_{\mathrm{MSD}}$ (indicated with red arrows), appear in clusters. If we now visualize the motions of these clusters, we find that for $\zeta=$ 3.4, their motion and thus the cage rearrangements promote motion in the circumferential direction, while this motion is not directed for $\zeta=3.8$. To further quantify this, we plot in Fig. 3(d) the absolute value of the angle of the displacement vectors, relative to the $y$-axes for red (mobile) particles, which have $\Delta r>2 \times \Delta r_{\mathrm{MSD}}$, and grey (immobile) particles, which have $\Delta r<2 \times \Delta r_{\text {MSD }}$. Particularly for $\zeta=3.4$, where we previously saw that the anisotropy is maximal, the slow particles do not show any directionality but the fast moving clusters - that dominate the diffusivity - markedly do so.

Based on previous work on dense suspensions under confinement, we also expect to see some intrinsic effects of the constraint as a result of a confinement dependent flow cooperativity, known to affect the diffusivity in narrow channels. ${ }^{11,12}$ In order to establish whether this is also at play here, we suppress the tendency to order by introducing polydispersity into the system. In Fig. 3(e) we plot the ratio $D_{\mathrm{C}} / D_{\mathrm{L}}$ as function of $\zeta$ for various degrees of polydispersity (blue curve: monodisperse, red curve: tridisperse (particle diameter: 0.9, 1.0, 1.1) and the black curve where we consider five different particle diameters $(0.8,0.9,1.0,1.1,1.2))$. We find that for increasing

(a)

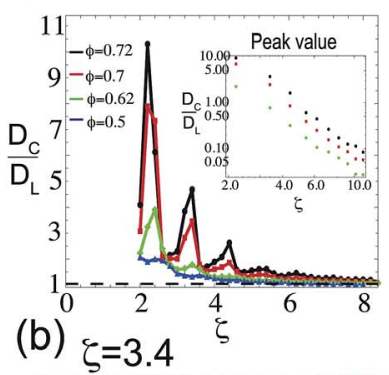

(c)
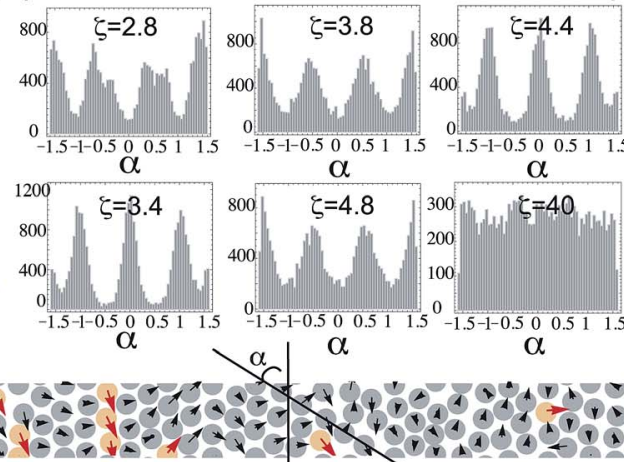

$\alpha$

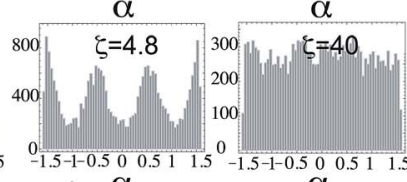

$\alpha$

(d)

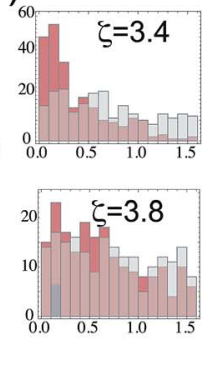

(e)
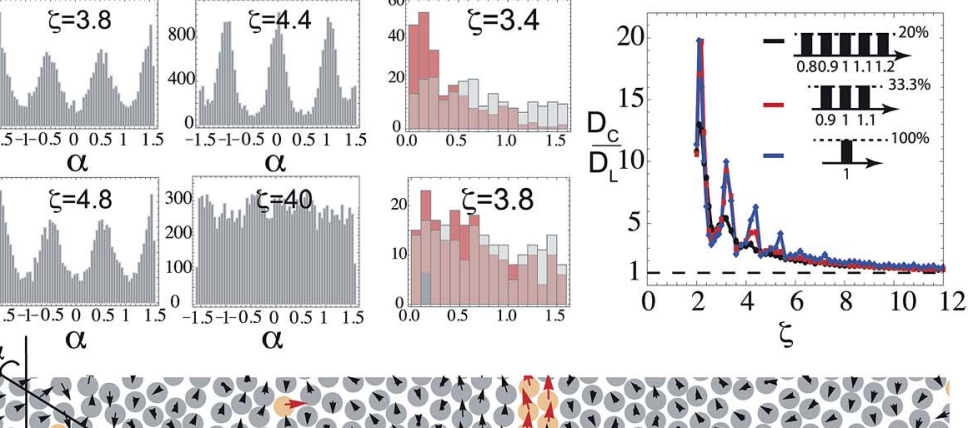

. $\zeta=3.8$

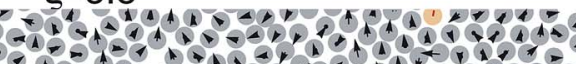

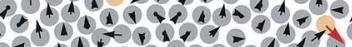
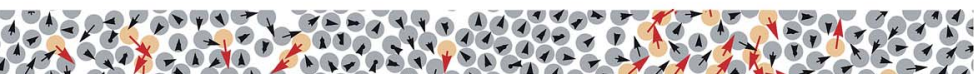

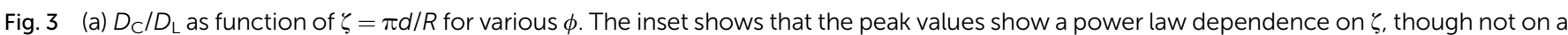

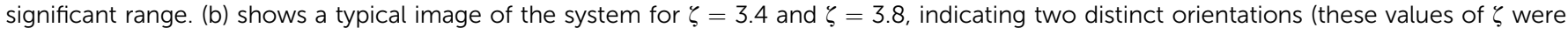

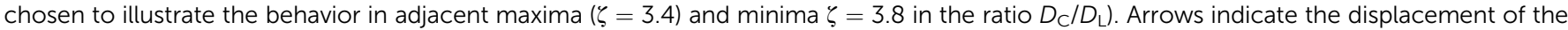

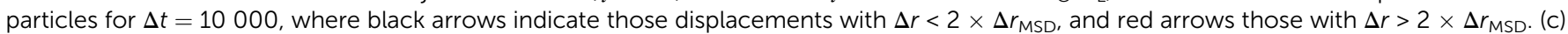

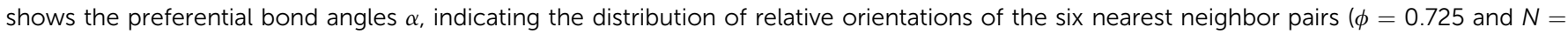

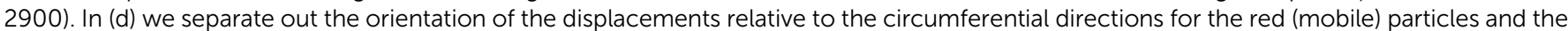

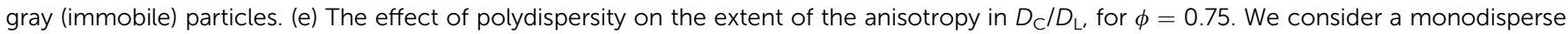
system (blue curve), a tridisperse system (red curve) and a polydisperse system (black curve). 
polydispersity the peaks in $D_{\mathrm{C}} / D_{\mathrm{L}}$ are reduced, confirming the configurational nature of the anisotropy, but that the increasing trend of $D_{\mathrm{C}} / D_{\mathrm{L}}$ for decreasing $\zeta$ remains. We speculate, that this may be related to a side effect of wall confinement in glassy flows, where fragile zones appear to live much longer near the wall than they do in the bulk. ${ }^{11,12}$ Even absent a physical wall, our systems are confined in the circumferential direction and we expect collective rearrangements in that direction to live longer than those along the long axis of the system.

To summarize, we have addressed the question of how the lateral diffusion of densely packed particles, confined to a cylinder, is affected by the shape of the substrate and the packing fraction. We find that the shape of the substrate in combination with crowding induces an anisotropy between the longitudinal and the circumferential diffusivity. We find that the extrema of the anisotropy coincide with an ordered packing of discs in a triangular lattice and its affinely rotated structure, geometries also observed for dense athermal packings. ${ }^{\mathbf{1 4}}$ These skewed patterns promotes collective motion in the circumferential direction.

Our work reveals that diffusive motion of discs along the surface of a narrow, crowded tube is hindered by an interplay between local geometry and crowding. Diffusivity in highly crowded systems is dominated by the collective motion of clusters and the size of these clusters may, especially at high coverage, become comparable to the local radii of curvature of the system. Though we focus on diffusion on cylindrical surfaces here of discs with radii comparable to the typical radius of curvature of the substrate, even the diffusion of discs or particles that are small compared to the local radius of curvature may very well be sensitively affected by the curvature of the substrate; not by their proper size, but through the size of the cage through which they diffuse, which - particularly at elevated densities - may become comparable in size to the local radius of curvature. From a more practical viewpoint, engineered structures such as colloids confined to an interface and topological emulsions are systems where curvature and crowding directly meet and would be ideal candidates to experimentally verify this geometrically induced anisotropic diffusion. Some specific biological settings where dense packings as well as high curvatures feature are cristae in mitochondria, dendritic spines in synapses and grana thylakoids in choroplasts, where the sizes of narrow membrane tubes, connecting these microdomains to the remainder of the cell, are not that much larger than the protein complexes that diffuse on it. In this work, we have focused on the effect of the shape of the substrate itself. We should note, however, that proteins diffusing along these structures are most likely not isotropic - not in size, nor in their interaction. We expect such anisotropies in protein features to considerably affect the ordering at higher densities, and thus to cause an additional anisotropy. Moreover, even a transmembrane protein with a perfectly cylindrical cross section in 3D would present an anisotropic footprint on a cylindrical section of membrane, deviating from the discs we consider. The computational tools to confine particles to more complex surfaces have recently been developed and will be employed in the near future to further study effects of particle shape, boundary and surface geometry. ${ }^{19}$

\section{Acknowledgements}

We thank Thijs Michels and Claus Heussinger for valuable discussions. This work was supported by funds from the Netherlands Organization for Scientific Research (no. FOME1012M) and the Human Frontier Science Program (no. RGP0017/2012).

\section{References}

1 F. Varnik, J. Baschnagel and K. Binder, Phys. Rev. E: Stat., Nonlinear, Soft Matter Phys., 2002, 65, 021507.

2 D. Lucena, D. Tkachenko, K. Nelissen, V. Misko, W. Ferreira, G. Farias and F. Peeters, Phys. Rev. E: Stat., Nonlinear, Soft Matter Phys., 2012, 85, 031147.

3 J. Mittal, T. M. Truskett, J. R. Errington and G. Hummer, Phys. Rev. Lett., 2008, 100, 145901.

4 S. Lang, V. Boțan, M. Oettel, D. Hajnal, T. Franosch and R. Schilling, Phys. Rev. Lett., 2010, 105, 125701.

5 S. Mandal, S. Lang, M. Gross, M. Oettel, D. Raabe, T. Franosch and F. Varnik, 2014, arXiv preprint arXiv:1406.5327.

6 H. C. Shum, A. R. Abate, D. Lee, A. R. Studart, B. Wang, C.-H. Chen, J. Thiele, R. K. Shah, A. Krummel and D. A. Weitz, Macromol. Rapid Commun., 2010, 31, 108-118.

7 D. Ershov, J. Sprakel, J. Appel, M. A. C. Stuart and J. van der Gucht, Proc. Natl. Acad. Sci. U. S. A., 2013, 110, 9220-9224.

8 R. Kusters, L. C. Kapitein, C. C. Hoogenraad and C. Storm, Biophys. J., 2013, 105, 2743-2750.

9 R. Kusters and C. Storm, Phys. Rev. E: Stat., Nonlinear, Soft Matter Phys., 2014, 89, 032723.

10 F. Höfling and T. Franosch, Rep. Prog. Phys., 2013, 76, 046602.

11 J. Goyon, A. Colin, G. Ovarlez, A. Ajdari and L. Bocquet, Nature, 2008, 454, 84-87.

12 L. Bocquet, A. Colin and A. Ajdari, Phys. Rev. Lett., 2009, 103, 036001.

13 A. Mughal, H. K. Chan and D. Weaire, Phys. Rev. Lett., 2011, 106, 115704.

14 A. Mughal and D. Weaire, Phys. Rev. E: Stat., Nonlinear, Soft Matter Phys., 2014, 89, 042307.

15 S. Plimpton, J. Comput. Phys., 1995, 117, 1-19.

16 N. Dan, P. Pincus and S. Safran, Langmuir, 1993, 9, 27682771.

17 M. M. Müller, M. Deserno and J. Guven, Phys. Rev. E: Stat., Nonlinear, Soft Matter Phys., 2005, 72, 061407.

18 S. Semrau, T. Idema, T. Schmidt and C. Storm, Biophys. J., 2009, 96, 4906-4915.

19 S. Paquay and R. Kusters, 2014, arXiv:1411.3019.

20 C. Donati, S. C. Glotzer, P. H. Poole, W. Kob and S. J. Plimpton, Phys. Rev. E: Stat. Phys., Plasmas, Fluids, Relat. Interdiscip. Top., 1999, 60, 3107-3119. 